\title{
Pharmacological pain management in the elderly patient
}

\section{Gary McCleane}

Rampark Pain Centre, Lurgan, Northern Ireland, United Kingdom
Correspondence: Gary McCleane Rampark Pain Centre, 2 Rampark Pain Centre, Lurgan BT66 7JH,

Northern Ireland, United Kingdom

Tel + I 442838349799

Fax + I 442838349799

Email gary@mccleane.freeserve.co.uk

\begin{abstract}
With the increasing number of elderly patients the issue of pain management for older people is of increasing relevance. The alterations with aging of the neurobiology of pain have impacts of pain threshold, tolerance and treatment. In this review the available evidence from animal and human experimentation is discussed to highlight the differences between young and older subjects along with consideration of how these changes have practical effect on drug treatment of pain. Cognitive impairment, physical disability and social isolation can also impact on the accessibility of treatment and have to be considered along with the biological changes with ageing. Conventional pain therapies, while verified in younger adults cannot be automatically applied to the elderly without consideration of all these factors and in no other group of patients is a holistic approach to treatment more important.
\end{abstract}

Keywords: pain, analgesia, pain threshold, pain tolerance

There is little merit in considering the treatment of pain in elderly patients as a separate entity unless there are real and practical differences to its management in younger subjects. Few would argue that these differences do not exist although the definition of when a patient becomes "elderly" is problematical. We all know of "young" patients who are physiologically and medically old, while others, although chronologically advanced retain the health and constitution of others decades younger.

Clearly many disease states become more common as age advances. These conditions may cause acute illnesses that need operative intervention (leading to pain) or may cause more chronic pain related disability. But it is about more than just the physical health of the patient. We can see the physiological parameters and results of blood screening as we measure them and make alterations to our treatment plans for that patient, but can easily forget the other issues that contribute to holistic care. The elderly are more likely to have sensory impairments, memory difficulties and lack of social backup that can all interfere with treatment and illness recovery. As educated practitioners it is easy for us to suggest treatment which may well have the potential for being effective with that patient. However, the patient cannot read the drug label which states the dose and dose frequency, cannot open the container because of arthritic hands, and cannot get assistance with drug administration because they live on their own. Therefore, an awareness of all those factors which accompany ageing is significantly more important than just the knowledge of how pain drug treatments differ in the elderly.

As well as increased incidence of illness with advancing years, problems of communication with older patients can arise. Impairment of sight and hearing can have obvious consequences. Even more problematical is the patient with dementia. They suffer pain as much as anyone else but may lack the cognitive function that allows them to communicate their pain or the response to drug intervention. Their reaction 
to pain may be to exhibit abnormal behaviour which can be misconstrued as worsening mental function or aggression. When dealing with the intricacies of pain diagnosis, the inability of the patient to describe the location, duration and characterization of their pain can make the diagnostic process infinitely more difficult with obvious repercussions on the quality of treatment offered. As with young children, imaginative methods of assessing pain may aid correct interpretation and treatment.

Allied to all of this is the issue of accessibility to treatment. In terms of chronic pain, the vast majority of pain clinics are Hospital based and a proportion of the treatments they offer are available only in a Hospital environment. With the physical disability that can accompany ageing, accessibility of these services may be less easy. Perhaps what we need is an increased understanding in the Primary Care environment that would enable effective treatment to be instituted at home and not necessitate Hospital referral.

\section{An aging population}

In the year 2000, there were more than 400 million people aged 65 and over in the world with a projected increase to almost 1.5 billion by the year 2050 (US Bureau of the Census 2002). This represents a fourfold increase compared to the 50 percent increase for the global population as a whole (Lunenfeld 2002). By that time, it is expected that $25 \%$ of these elderly people will be over the age of 80 . Epidemiological evidence suggests that acute pain is similar across all age groups (Corran et al 1997; Helme and Gibson 2002). Chronic pain seems to increase in incidence up to the seventh decade, although the nature and type of this pain may differ from that found in younger people. Naturally, older patients are more likely to suffer pain as a result of degenerative diseases, as compared to those of less advanced years.

Rustoen et al (2005) mailed 4000 Norwegian citizens with a pain and health questionnaire. $19.2 \%$ of the $18-39$ year old group reported pain of greater than 3 months duration, $27.5 \%$ of the $40-59$ year olds also reported chronic pain while the figure was $31.2 \%$ in the $60-81$ year old group. Therefore, in this population group, chronic pain was more frequent not only in the old, but also in the middle age group when compared to younger adults.

\section{Neural differences in the aged}

Animal studies suggest that ageing has a significant effect on aspects of the morphological and functional operation of the peripheral nervous system (Khalil et al 1994). A decrease in the major myelin proteins contributes to a loss in myelinated and unmyelinated nerve fibers in the elderly. Axonal atrophy is more common observed (Verdu et al 2000) while nerve conduction and endoneural blood flow are reduced with advancing age contributing to a reduction in peripheral nerve function (Verdu et al 2000). Even when regeneration of damaged neurons occurs, these regenerated fibers have a smaller number of terminal and collateral synapses.

In aged rats, immunochemical studies of the spinal cord reveal an increase in mRNA content of the neuropeptide tyrosine and galanin in dorsal root ganglia (DRG) neurons. These animals have decreased cellular content of calcitonin gene-related peptide and substance $\mathrm{P}$ compared to younger animals, while their levels of somatostatin are similar. The labeling intensity for encoding high-affinity tyrosine receptors (TrkA, Trk B and Trk C) are decreased in the DRG neurons of the aged rats (Bergman et al 1996).

It has also been noted that there is a progressive loss of both serotinergic and noradrenergic neurons in the superficial lamina of the spinal dorsal horn (Wong et al 1984, Laporte et al 1996). These neurons are closely implicated in descending bulbospinal inhibitory control, and such loss may upset the natural endogenous pain-suppressing mechanisms.

At supraspinal levels, there is reduced neurotransmitter content expression (Barili et al 1998), decreased metabolic turnover and a loss of neurons and dendritic connections throughout the cerebral cortex, midbrain and brainstem (Pakkenberg and Gundersen 1997). It is inevitable that such differences result in a differing incidence, quality and hence treatment needs of older patients as compared to younger adults.

\section{Effect of age in animal pain models}

It is known that levels of the neurotransmitter substance $\mathrm{P}(\mathrm{SP})$ in the spinal cord are lower in very old rats when compared to that in young rats. After peripheral nerve injury, immunoreactivity to the SP receptor (NK 1, neurokinin 1 receptor) increases in the spinal cord ipsilateral to the injury and the increase correlates to the development of thermal hyperalgesia (Cruce et al 2001).

In rats having an incision inflicted on one of their paws and the consequent mechanical sensitivity and thermal responses tested, both young and old rats respond in a similar fashion to the thermal stimulus. However, younger animals appear to recover more quickly from the mechanical allodynia produced by the paw incision when compared to the older rats (Ririe et al 2003). This suggests that modulation of A-fiber mediated sensitization differs in young and old rats. 
Studies on isolated spinal cord neurons show that spontaneous firing rates is higher and the response to thermal stimulation are greater in aged as compared to adult rats (Chakour et al 1996). Furthermore, the size of the receptive field area of wide-dynamic range neurons is larger and that of low-threshold neurons smaller in aged as compared to adult rats. The increased nociceptive neuronal activity in older rats correlates with the finding that paw withdrawal latency is significantly shorter in aged as compared to adult rats following heat stimulation of the paw. This along with the previously mentioned loss of serotinergic and noradrenergic fibers in the spinal dorsal horn of older rats may contribute to the apparent diminution of descending inhibitory control of nociceptive processing in older animals.

Pickering et al (2006) studied young, old and senescent rats using a chronic sciatic nerve constriction model. They found that senescent animals were less sensitive to neuropathic pain than old or young rats while these senescent animals were more sensitive to acute pain than the other groups. All of these findings confirm that from a physiological perspective, definite differences exist between young adult and older animals.

So animal responses to pain stimuli may differ when adult and aged animals are examined. So too may drug effects. We are currently seeing an increasing use of opioids, and in particular strong opioids, in the management of chronic pain. Analgesic tolerance is a potential complication of such opioid use. In rat models tolerance is easy to demonstrate. Wang et al (2005) examined rats aged 3 weeks, 3, 6 and 12 months. Morphine was injected twice daily and the time until analgesic tolerance measured (which they defined as a $75 \%$ reduction in morphine-induced analgesia compared to day (1). Tolerance was apparent on the 4th, 10th, 14th and 22nd days of morphine treatment, respectively. Plasma morphine levels and that of its metabolites did not correlate with the differences in tolerance development. One other factor that impinges on pain perception is the hormonal status of the patient. We have seen that in animal models that advancing age does have a noticeable effect. But, particularly in the female, age brings about hormonal changes as well so that, for example, response to opioid analgesics is similar in males to gonadectomized and reproductively senescent females but differs to that of reproductively active females (Sternberg et al 2004).

\section{Effect of age on human pain models}

It is not easy to equate the results of human experimental pain tests with actual clinical pain conditions. Experimental pain tests examine single modalities of pain whereas in clinical pain practice a noxious stimulus often produces pain which has different facets.

Perhaps the most clinically relevant issues are around those of pain threshold and pain tolerance. Gibson (2003) reported that over 50 studies have examined age differences in sensitivity to experimentally induced pain and that the majority of these studies have focused on pain threshold. Twenty one studies reported an increase in pain threshold with advancing age, 3 a decrease, and 17 no change. When all results are examined meta-analytically, the effect size is 0.74 ( $p<0.0005)$, indicating that there is definite evidence of an increase in pain threshold with advancing age. That said, there may well be a difference in pain threshold depending on the type of pain inflicted. Lautenbacher et al (2005) studied 20 young men with an average age of 27.1 years and compared then to 20 elderly men with an average age of 71.6 years. They found that somatosensory thresholds for non-noxious stimuli increased with age whereas pressure pain thresholds decreased and heat pain thresholds showed no age-related changes. These results are confirmed by Lin and colleagues (2005) who found that there was a consistent relationship between sensory thresholds as measured by quantitative sensory testing and age.

When pain tolerance is considered, the 10 studies examining the effect of age on pain tolerance show a definite age-related decrease in willingness to endure very strong pain. The decrease in pain tolerance is estimated at -0.45 $(\mathrm{p}<0.001)$ across these studies.

Lasch et al (1997) examined the effect of intraesophageal balloon dilatation in healthy young and older adults. The volume of air inflated into the balloon before report of pain was measured. The volume was significantly higher in the older subjects. Indeed, many of the older subjects failed to report pain even after maximal balloon inflation, in marked contrast to the younger patients. It certainly seems, using this experimental technique, pain threshold does increase with age. But this study also reminds us that pain is not always an entirely negative symptom. On many occasions it provides timely warning of impending problems. For example, if it requires a larger volume within a hollow viscus in an elderly patient before pain is experienced as compared to younger subjects, then the discomfort caused by an obstructive lesion in the bowel, for example, may take longer to become symptomatic in older patients, with all the consequences for the treatment and prognosis of the causative lesion. It is also clinically apparent that the incidence of silent myocardial ischemia increases with age (Lucantoni et al 1997). Whether 
this is due to an increased pain threshold in the older patient, a pain reducing effect of concomitant medication, or the effects of an intercurrent illness is debatable, but all probably have some effect. Therefore, while our emphasis is on the management of pain in the elderly, we should not lose sight of the fact that thought must also be given to the absence of pain in these patients under circumstances where it would normally be present.

\section{Pharmacokinetic alterations}

Physiological changes in older people affecting fat mass (increased), muscle mass (reduced), and body water (reduced) have important effects on drug distribution (Turnheim 2003; Vuyk 2003). Blood volume may also be reduced due to concomitant use of diuretics. With the reduction in relative fat mass, those drugs which are highly lipophylic, such as fentanyl and lidocaine, can have an increased duration of effect as less of them are soaked up by fat tissue. In contrast water soluble dugs, such as morphine) are less efficiently distributed and higher plasma concentrations are obtained with equivalent doses and so side effects may be more frequent.

Free drug availability is significantly augmented by decreases in serum albumin, particularly in those elderly patients with chronic disease and malnutrition. Such changes increase the potential for adverse effects associated with highly protein bound analgesics such as NSAIDs and antiepileptic agents such as valproate, phenytoin and carbamazepine. Levels of alpha 1-acid glycoprotein, the serum carrier for basic drugs such as meperidine, appear unchanged in older people.

With increased age, liver and kidney function decrease and they become progressively less efficient at drug clearance. Drug half-life, a ratio of the volume of distribution to clearance, is notably increased for several benzodiazepine and tricyclic antidepressants.

Lipid-soluble drugs such as lidocaine and narcotic analgesics are good examples of drugs that undergo significant firstpass metabolism during passage from the gastrointestinal tract to the liver. Peak plasma concentrations may rise as may the potential for dose-related side effects. This situation is further compounded when cardiac output is impaired through disease. Highly protein bound drugs are less influenced by first-pass metabolism (Bresler and Bahl 2003).

It seems that hepatic phase I reactions involving oxidation, hydrolysis and reduction appear more strongly altered by age than phase II conjugation processes (acetylation, glucuronidation, sulfation, and glycine conjugation). In general, phase I reactions diminish irrespective of which microsomal cytochrome P450 enzyme is involved, although interindividual variation can be significant. Acetaminophen and diazepam, both processed through the CYP3A4 and 3A5 enzyme routes, are metabolized at equal rates irrespective of age. Carbamazepine, lidocaine and fentanyl, in contrast, are subject to reduced metabolism by the same enzyme systems in older patients. Glucuronidation of morphine and glutathione conjugation of acetaminophen are examples of reduced and unaltered phase II reactions respectively. Age appears to have no effect on the frequencies of slow- and rapid metabolizing genetic polymorphisms.

The most important pharmacokinetic effect of age is the reduction in renal clearance. This can be compounded by illnesses which further reduce renal function. This can lead to drug toxicity at dosing levels appropriate for a younger patient with normal renal function. Drugs with a predominant renal mode of excretion, such as gabapentin, can cumulate when kidney function fails.

\section{Pharmacodynamic changes}

Whether the elderly demonstrate actual changes in intrinsic sensitivity at the receptor level is controversial both in terms of measurable alterations in receptor numbers and the efficiency of signal transduction following receptor binding. It has been shown that in elderly rats, the number of mu and kappa opioid receptor numbers fall, while delta-opioid receptor numbers stay unchanged.

One factor that is of practical concern is that of analgesic tolerance when opioid drugs are being used. Buntin-Mushock et al (2005) performed a retrospective chart review of 206 patients who had been prescribed strong opioids. They found that younger patients reached a maximum dose of around $450 \mathrm{mg}$ of oral morphine over a 15 month period, while the older patients achieved a maximum dose of around $210 \mathrm{mg}$ over 14 months. When discharged from their clinic only the older patients actually had a reduction in the visual analogue pain scores. They suggest that perhaps older patients have less chance of developing opioid analgesic tolerance. From a practical perspective, decline in homeostatic counter-regulatory mechanisms in older patients create a less forgiving background to drug administration and it is clear that older patients are less able to regain their original physiological steady state after administration of drugs. For example, tricyclic antidepressants and opioids can induce orthostatic hypotension and precipitate falls and syncope. Homeostatic changes increase the risk of gastric irritation and bleeding following exposure to NSAIDs and figures would suggest 
that the risk of gastric bleeding is four times higher in older individuals when compared to younger adults.

\section{Effect of age on human clinical pain}

Clinical observation would suggest that there is an increased incidence of silent myocardial ischemia in elderly patients and this exemplifies the fact that advancing age has an effect on pain appreciation. Another example would be the atypical presentation of an inflamed appendix where there may be an absence of localized right iliac fossa pain. Differences in the neuroanatomy, physiology and biochemistry of the nociceptive pathways may cause alterations in pain perception while differences in the pharmacology of drugs in the elderly may alter expected responses to these drugs. Further compounding these issues is the perception of pain in elderly patients by their physicians. Yunus et al (1988) compared elderly and young patients with fibromyalgia. They found that chronic headaches, anxiety, tension, mental stress and poor sleep were all les common in elderly patients with this condition. But most importantly, in only $17 \%$ of the elderly patients in the study were the features of fibromyalgia recognised initially by their physician prior to Rheumatological referral. Many of these patients were exposed to inappropriate corticosteroid treatment in the mistaken belief that they had an inflammatory condition.

The magnitude of the problem of pain in elderly patients is highlighted by figures from Sweden. Here a survey revealed that $73 \%$ of those older that 77 years had pain while $68 \%$ of those older than 85 years also suffered with pain (Bratberg et al 1996). The incidence of pain in the elderly patient is affected by a range of variables which include racial and ethnic susceptibility to chronic disease, socioeconomic factors and to accessibility to health care (Reyes-Gibby et al 2007).

When one tries to consider individual pain conditions then there is a lack of depth of evidence on which to base conclusions. For example, in the case of low back pain, when consideration is given to evidence in published studies then there is a marked under-representation of studies that include elderly patients and so firm conclusions cannot be reached (Bressler et al 1999; Dionne et al 2006). It is however clear that when pain does occur in elderly patients it impacts on the overall quality of life of the individual (Salaffi et al 2005). Analgesic treatment is therefore desirable remembering that the response of elderly patients to drug therapy may differ to that of younger individuals. It may be that elderly patients require lower dosing of opioid analgesics, have a more rapid response to them and develop analgesic tolerance more slowly than those of less advanced years (Buntin-Mushock 2005).

Along with aging comes and increased incidence of conditions such as Alzheimer's disease. It has been observed that the use of non-steroidal anti-inflammatory drugs is lower in patients with this condition than those of a similar age without it, and the use of anti-inflammatories is not dependent on the stage of the Alzheimer's disease (Scherder and Bouma 1997). When patients with Alzheimer's disease and age matched controls are again considered, those with acute pain consume a similar quantity of pain killers to non-Alzheimer's sufferers, and it is only in those with chronic pain that consumption is significantly lower (Pickering et al 2006). In contrast, patients with vascular dementia seem to suffer more pain than others without cognitive impairment due to this condition (Scherder et al 2003). As well as differences in pain experience and analgesic use, cognitive impairment can also lead to a significant under-reporting of pain related conditions. Indeed Corali et al (2006) found that $41.4 \%$ of patients admitted to a Geriatric Evaluation and Rehabilitation Unit had previously undiagnosed osteoarthritis.

Overall, therefore, as age advances the incidence of pain causing conditions differs to that of younger patients, impact on quality of life may be greater and a differing response to analgesic intervention may be observed.

\section{Drug treatment}

An increasing number of consensus statements are being published by professional groups suggesting pharmacological strategies for providing pain relief. While these represent a useful formalization of available evidence, they have a strong tendency to suggest medications which have a definite indication for the suggested use. For example, when neuropathic pain is considered, gabapentin and pregabalin are prominent suggested treatments. These recommendations are based both on the fact that they have product licenses for us in neuropathic pain and because there is significant evidence of effect in the general, but not necessarily the elderly, population. If patients ability to tolerate drugs decreases and anxieties about potential drug-drug interactions increases with advancing years, then consideration may need to be given to "off-label" use of drugs. When a specific indication is sought for a drug, the licensing authorities base their considerations on three primary factors. First the physical quality of the preparation. Second its safety and third the evidence of its effect in that condition. There are, however, many drugs successfully used in pain management whose use is "off-label" because the practitioner considers the risk to the patient to be less and the 
chances of alleviating pain greater when that drug is used as opposed to a licensed alternative.

For example, osteoarthritis is extremely common in elderly patients. Conventional treatment would involve the use of non-steroidal anti-inflammatory drugs. These may be contraindicated in some elderly patients because of heart failure, renal failure, concomitant drug use or because of previous peptic ulcer disease. Topical nitrates can produce marked reduction in inflammation and pain (McCleane 2000a, 2007b). Similarly, amitriptyline is often prescribed for neuropathic pain conditions such as postherpetic neuralgia. Topical tricyclic antidepressants can also have a pain reducing effect without the common side effects associated with oral tricyclic use (McCleane 2000b, 2007). It may well be that the practitioner has to make a valued judgment about whether it is in the best interests of the patient to use "conventional", licensed medication, or because of anxieties about the patients state of health resultant in their advanced age as to whether "off-label" use of drugs with proven pain relieving effect may be more appropriate.

\section{Conclusions}

Experimental testing in both animals and humans, and indeed practical experience confirm that the experience of pain, its presentation, diagnosis, pathophysiology and actual treatment change as age progresses. In one sense the chronological age of the patient is not relevant as it gives an artificial estimate of the individual's health status. What is more important is how that individual's health deviates from what may be considered average for an adult. In anesthesiological practice, for example, the risk of a patient undergoing anesthesia is not estimated using age as a criterion. Rather an estimate of risk is made using assessment scales, such as the "ASA" grading system which classify the patient's health using the presence or absence of disease states, whether these diseases are mild or severe and whether they represent a constant threat to life.

It could be argued, therefore, that one should not base a diagnosis and plan treatment on the basis of that patient's age alone. For all patients, regardless of age, we should have an individual approach and construct a diagnostic package and treatment plan based on that individual's situation, taking account of their physical and mental health, expectations, social circumstances and their general well being.

\section{References}

Barili P, De Carolis G, Zaccheo D, et al. 1998. Sensitivity to ageing of the limbic dopaminergic system: a review. Mech Ageing Dev, 106:57-92.
Bergman E, Johnston H, Zhang X, et al. 1996. Neuropeptides and neurotrophin receptor mRNAs in primary sensory neurons of aged rats. J Comp Neurol, 11:303-19.

Bontin-Mushock C, Philip L, Moriyama K, et al. 2005. Age dependent opioid escalation in chronic pain patients. Anesth Analg, 100:1740-5.

Bresler R, Bahl JJ. 2003. Principles of drug therapy for the elderly patient. Mayo Clin Proc, 78:1564-77.

Bressler HB, Keyes WJ, Rochon PA, et al. 1999. The prevalence of low back pain in the elderly. A systematic review of the literature. Spine, 24:1813-9.

Brattberg G, Parker MG, Thorslund M. 1996. The prevalence of pain among the oldest in Sweden. Pain, 67:29-34.

Chakour MC, Gibson SJ, Bradbeer M, et al. 1996. The effect of age on A delta and C fiber thermal pain perception. Pain, 64:143-52.

Corali C, Franzoni S, Gatti S, et al. 2006. Diagnosis of chronic pain caused by osteoarthritis and prescription of analgesics in patients with cognitive impairment. J Am Med Dir Assoc, 7:1-5.

Corran TM, Farrell MJ, Helme RD, et al. 1997. The classification of patients with chronic pain: age as a contributing factor. Clin J Pain, 13:207-14.

Cruce WL, Lovell JA, Crisp T, et al. 2001. Effect of aging on the substance $P$ receptor, NK-1 in the spinal cord in rats with peripheral nerve injury. Somatosens Mot Res, 18:66-75.

Dionne CE, Dunn KM, Croft PR. 2006. Does back pain prevalence really decrease with increasing age? A systematic review. Age Ageing, 35:229-34.

Gagliese L, Melzack R. 2000. Age differences in nociception and pain behaviour in the rat. Neurosci Biobehav Rev, 24:843-54.

Gibson SJ. 2003. Pain and aging: the pain experience over the adult lifespan. In Proceedings of the 10th World Congress on Pain, IASP Press, Seattle, 2003.

Helme RD, Gibson SJ. 2001. The epidemiology of pain in elderly people. Clin Geriatr Med, 17:417-31.

Khalil Z, Ralevic V, Bassirit M, et al. 1994. Effects of ageing on sensory nerve function in rat skin. Brain Res, 641:265-72.

Laporte AM, Doyen C, Nevo IT. 1996. Autoradiographic mapping of serotonin 5HT1A, 5HT1D, 5HT2A and 5HT3 receptors in the aged human spinal cord. J Chem Neuroanat, 11:67-75.

Lasch H, Castell DO, Castell JA. 1997. Evidence for diminished visceral pain with aging; studies using graded intraesophageal balloon distension. Am J Physiol, 272:G1-3.

Latienbacher S, Kunz M, Strate P, et al. 2005. Age effects on pain thresholds, temporal summation and spatial summation of heat and pressure pain. Pain, 115:410-18.

Lucantoni C, Marinelli S, Refe A, et al. 1997. Course of pain sensitivity in aging: pathogenetical aspects of silent cardiopathy. Arch Gerentol Ger, 24:281-6.

Lin YH, Hsieh SC, Chao CC, et al. 2005. Influence of aging on thermal and vibratory thresholds of quantitative sensory testing. J Peripher Nerv Syst, 10:269-81.

Lunenfeld B. 2002. The ageing male: demographics and challenges. World J Urol, 20:11-16.

McCleane GJ. 2000a. The analgesic efficacy of topical capsaicin is enhanced by glyceryl trinitrate in painful osteoarthritis: a randomized, doubleblind, placebo controlled study. Eur J Pain, 4:355-60.

McCleane GJ. 2000b. Topical application of doxepin hydrochloride, capsaicin and a combination of both produces analgesia in human neuropathic pain: a randomised, double-blind, placebo controlled study. Br J Clin Pharmacol, 49:574-9.

McCleane GJ. 2007a. Topical analgesics. Medical Clinics North America, 91:125-39.

McCleane GJ. 2007b. Topical analgesic agents. Medical Clinics in Geriatric Medicine, In Press.

Pakkenberg B, Gundersen HJ. 1997. Neocortical neuron numbers in humans: effects of sex and age. J Comp Neurol, 384:312-20.

Pickering G, Jourdan D, Millecamps M, et al. 2006. Age-related impact of neuropathic pain on animal behaviour. Eur J Pain, 10:749-55. 
Ririe DG, Vernon TL, Tobin JR, et al. 2003. Age dependent responses to thermal hyperalgesia and mechanical allodynia in a rat model of acute postoperative pain. Anesthesiology, 99:443-8.

Reyes-Gibby CC, Aday LA, Todd KH, et al. 2007. Pain in aging communitydwelling adults in the United States: non-Hispanic whites, non-Hispanic blacks, and Hispanics. J Pain, 8:75-84.

Rusten T, Wahhl AK, Hanestad BR, et al. 2005. Age and the experience of chronic pain: differences in health and quality of life among younger, middle-aged, and older adults. Clin J Pain, 21:513-23.

Salaffi F, Carotti M, Stancati A, et al. 2005. Health-related quality of life in older adults with symptomatic hip and knee osteoarthritis: a comparison with matched healthy controls. Aging Clin Exp Res, 17:253-4.

Scherder EJ, Bouma A. 1997. Is decreased use of analgesics in Alzheimer disease due to change in the affective component of pain? Alzheimer Dis Assoc Disord, 11:171-4.

Scherder EJ, Slaets J, Deijen JB, et al. 2003. Pain assessment in patients with possible vascular dementia. Psychiatry, 66:133-45.

Sternberg WF, Ritchie J, Mogil JS. 2004. Qualitative sex differences in kappa-opioid analgesia in mice are dependent on age. Neurosci Lett, 363:178-81.
Turnheim K. 2003. When drug therapy gets old: pharmacokinetics and pharmacodynamics in the elderly. Exp Gerontol, 38:843-53.

US Bureau of the Census. 2002. International Data Base, Vol 2002. Washington DC: US Department of Commerce, US Bureau of the Census.

Verdu E, Ceballos D, Vilches JJ, et al. 2000. Influence of age on peripheral nerve function and regeneration. J Peripher Nerve Syst, 5:191-208.

Vuyk J. 2003. Pharmacodynamics in the elderly. Best Prac Res Clin Anaesthesiol, 17:207-18.

Wang Y, Mitchell J, Moriyama K, et al. 2005. Age-dependent morphine tolerance development in the rat. Anesth Analg, 100:1733-9.

Wong DF, Wagner HN, Dannals RF. 1984. Effects of age on dopamine and serotonin receptors measured by positron tomography in the living brain. Science, 226:1393-6.

Yunus MB, Holt GS, Masi AT, et al. 1988. Fibromyalgia syndrome among the elderly: comparison with younger patients. J Am Geriatr Soc, 36:987-95. 
